\title{
Space Scheduling for Construction Progress Planning and Control
}

\author{
I.D. Tommelein ${ }^{a}$ and P. P. Zouein ${ }^{b}$
}

a, b Civil and Environmental Engineering Department, The University of Michigan, Ann Arbor, MI 48109-2125, USA

\begin{abstract}
Space scheduling pertains to allocating work space to resources associated with activities in a schedule as it changes over time. This is an important aspect of materials management in dynamic environments in which many pieces of equipment, crews, and possibly robots move about, as congestion and interference would seriously hamper production. Advance space scheduling is then warranted to reduce the need for real-time sensing and adjusting. Reactive space scheduling may be needed when problems have arisen and must be alleviated. In any case, space-schedule data must be madel for dynamic layout fashion to all parties who need it. The MovePlan monce of space schedulinout planning is described to illustrate the overall significance of space scheduling to construction progress planning. MovePlan uses a two-dimensional representation of space to keep the computational costs associated with space-time calculations low. Accordingly, the model does not require a powerful workstatio a laptop computer-and is expected to run on a palmtop-that could easily be taken out into the field. MovePlan has been loosely integrated with a spaceschedule conflict resolver, named ConRes, which delays activities or changes their resource needs to lower the demand for space in so-called problematic time intervals. Issues pertaining to data collection for space scheduling, scheduling progress control, and data dissemination are discussed. A futuristic scenario is presented to illustrate the use of palmtop computing for space scheduling on site.
\end{abstract}

\section{INTRODUCTION}

In the last decade, microcomputer development has progressed tremendously and computer hardware costs have plummeted. Personal computing devices are now ubiquitous in construction home offices and on construction sites.

Several site materials management programs, including those described in this paper, are currently being developed to run on networked laptop computers. While such programs exhibit many useful features, they are limited in use due to the hardware on which they run. To make on-site computing become reality, wireless communication is a necessity and robust hardware must be dust, impact resist the harsh site conditions (e.g., large temperature gradients, dust, impact, and variable weather) under which construction is performed. 
automatically-generated solution. The development of interactive, heuristic decision support tools to aid them with dynamic layout planning and space scheduling is therefore advocated.

It is generally the case that construction schedules are too abstract (esp. executive-level milestone- and work package-, but even task-level schedules), i.e., they do not represent enough detaments can be studied early on in the planning process. Not enough decisions have been made at that time to allow for detailed space schedule development. Sufficient detail at the process level must be available (e.g., during daily crew-level planning) to watial and temporal data scheduling. Accordingly, it is at the procesividual workers and crews to organize must be collected and used to enable ind their progress. The term progress planning is used to denote this.

\section{SPATIAL AND TEMPORAL DATA}

\subsection{Data Collection}

The collection of large amounts of real-time field data has been made possible by the development of new tools such as laser-controlled and bar-coding systems. Laser-controlled systems assist in positioning the blades of grading equipment. This benefits individual equipment operation. Bar-coding of construction components (e.g., Bell and McCullouch 1988, Rasdorf and Herbert 1990a, 1990b) facilitates component identification and inventory status tracking. This benefits site management (performing tasks such as purchasing, inventory control, and site management (performing tasks such as purchature as to how these tools and
accounting), but there is little mention in the literature
the collected data can be used to improve crew-level operations and construction planning overall.

Data needed at the crew level should provide information to individual workers or superintendents when they are planning or executing work. It should provide answers to questions such as:

Where is the formwork?

Have the windows already been delivered? When will they arrive?

What is the nearest supply of two-by-fours?

Could I use the sheet rock that is over there? What will it be used for?

What is the best way to get to the management trailer?

Could I use that forklift to move the brick pallets closer to my work area?

Will that area be auailable tomorrow to set up a fabrication shop?

Clearly, much of the data needed to answer these questions changes over time. Materials may be brought to the site by one person, who needs to inform others of their arrival. Supplies get replenished during the course of the day, so workers who are waiting for them must be readily informed of the varies as work The nearest supply depends on where and. A material may appear to be available progresses and that worker moves designated for sole use by others. Congested to one worker, but mark must be circumvented if possible. Utility equipment and dangerous work apeally, it does not get scheduled; what appears to be idle at one time, may be tied up later. Crews need to communicate their plans to avoid future competition for resources or interference. Not only is it necessary to make 
The further decrease in size from laptop to palmtop computers, together with the standardization of wireless communication protocols, wajor impact on management techniques. Their combination is of such low-cost devices, the data field productivity. In anticipation be re-engineered to improve data collection, needs of field personnel scheduling, control, as well as data dissemination. Specifically, the planning and scheduling, be on characterizing construction resources in terms of focus of attention should the space they require and the timing of their presence their handling, including et al. 92). Research must investigate new methods to on site (Tommele resources tied to site space needs and scheduled time to effectively model resourials management systems (Bell et al. 87, Bell and Stukhart 86, 87; Lundberg and Beliveau 89).

\section{TERMINOLOGY}

When space is limited, tradeoffs between activity sequencing, timing, method selection, resource allocation, and space allocation are necessary to plan executable schedules and feasible layouts. Whe and reach of selected hoisting tradeoffs exists as, for example, travel distancy. The resource space as a variable equipment affect materials handing not been modeled with much detail, possibly in the scheduling process of the interaction between spatial and temporal aspects of resource needs and availability. To define the possible degree of interaction, two terms are used:

Dynamic layout planning (Tommelein and Zouein 93) refers to the unidirectional interaction between the scheduling and space allocation tasks. be final. It serves as input to the space allocation task.

Layouts can then be constructed for selected time intervals, each layout using the resources that are scheduled to be present during the interval it spans. It may be that no feasible layout can be constructed to acconing problem cannot be in each interval. In that case, the dynamic layou the schedule was frozen.

solved, possibly - but not necessarily Tommelein 93) refers to the bidirectional

Space scheduling (Zouein and and space allocation tasks. (Dynamic layout interaction between the scheduling scheduling.) A sequence of activities must be planning is thus a subtask of spacensions associated with each activity, and space defined, resources with their dimen resource. Schedule construction is performed on site must be identified for each rion. When layouts are found to be infeasible, concurrently with layout alter the problem so that it becomes solvable. For schedule changes might be delayed or have their resource use level changed to example, activities can duration.

Dynamic layout planning and space scheduling both are limited resource allocation problems involving the inherently two- (actually three-) dimensional variable space in addition to the scalar variables equallocation problems can be time, and possibly money. In general, limited restion techniques exist. This is one solved using heuristic methods only, nuch models conduct what-if analyses and solved using heuristic meth people who use such models conduct what-if anation an
experiment with alternate problem formulations, before accepting an 
spatial data available in a timely fashion to all people who need it; logging data as it changes over time must be done by all (or at least many) people on site as it is beyond the scope of what a single individual can do.

\subsection{Planning and Scheduling}

Few tools, beyond databases and traditional CPM programs, are currently available to aid with materials management as a true construction scheduling task. Construction materials come in great variety and arrive to the site under uncertain conditions. This poses challenges to management, beyond those of materials management in a steady-state manufacturing environment. The space resources require on site, their bulkiness, etc. may be a major hindrance in executing work according to plan. Managing materials accounts for a substantial part of construction cost, however, and research should investigate which models lend themselves to use on site.

\subsection{Progress Control}

Spatial data is particularly important at the process level. This is the level at which interferences can be prevented, excessive travel times identified, and appropriate material handling means selected. Three-dimensional CAD tools have been developed in recent years for checking interferences during the on-site movement and placement of exceptional loads. Little is formalized about the handling of most other materials on site, which are in the majority. The process level is also the level at which work progress can best be measured and hurdles overcome. It is therefore advocated that spatial data be included in control systems, which all too often limit themselves to modeling only durations and costs. An augmentation of simulation tools with spatial abstraction mechanisms might fill the need for tools to study the space-time-cost trade-off.

\subsection{Data Dissemination}

Materials data should be made available to all on site who need it. As different people have different data needs, appropriate abstraction mechanisms must be developed. Discussing these is beyond the scope of this paper. One issue worthwhile raising is that everyone on site will need a communication device to enter new and query existing data, but tight controls must be kept to guarantee data consistency and correctness. Wireless palmtop computers equipped with intelligent interfaces are expected to be able to meet those needs.

\section{ENVISIONED FIELD IMPLEMENTATION}

\subsection{Software Needs}

Few existing computer-based scheduling programs represent the resource space as an explicit variable. New software is therefore being developed to keep track of space schedules and to answer questions such as those mentioned in section 3. Our work includes three models, which address the space scheduling task with increasing degrees of complexity. 


\subsubsection{MovePlan}

Laying out temporary facilities on site all too often means to manually mark up a site arrangement drawing, showing different overlapping laydown areas drawing that can be intensecutive time frames. This results in a complicated means lends itself to updating only. by the one who made it, and that by no coordination and materials man as construction progresses. Its use for task

The MovePlan interas management is thus very limited.

Zouein 1993) exactly addresses this layout planning tool (Tommelein and schedule of activities annotated with throblem. MovePlan needs as input a rectangular space each resource will need on resources, the two-dimensional and resources on site, and the site's boundaries. It site, data describing the permanent time intervals of any length, related to parts. It enables users to create layouts for

The tool aids in solving this dynamic layout early-start project schedule. templates representing the resources that layout planning task by providing and a worksheet in which those templates are on site for the selected interval, is scaled to the site boundaries and shows can be moved around. The worksheet positioned resources are. When time-overlapping permanent and previouslymaintains consistency among them. A rapping layouts are created, MovePlan position in one layout, is shown in the A resource that has been assigned to a in time with the first one and that same position in any layout that overlaps schedule as soon as the first layout includes the resource. MovePlan freezes the layout sequence shows how the site nas been saved. A play-back of the dynamic provides decision support to field construction sites and coordino field engineers who arrange resources on

The MovePlan user may find that thetor or crew activities.

unsatisfactory (e.g., because find that the layout for a chosen time frame is critical resource). In this case, it may bested or no space is available to locate a resources in order to free up space for be possible to delay the arrival of some activities. Pertinent data must for others, or to assign different resources to makes the appropriate returned to MovePlan for the user to continue with the layout are subsequently

MovePlan

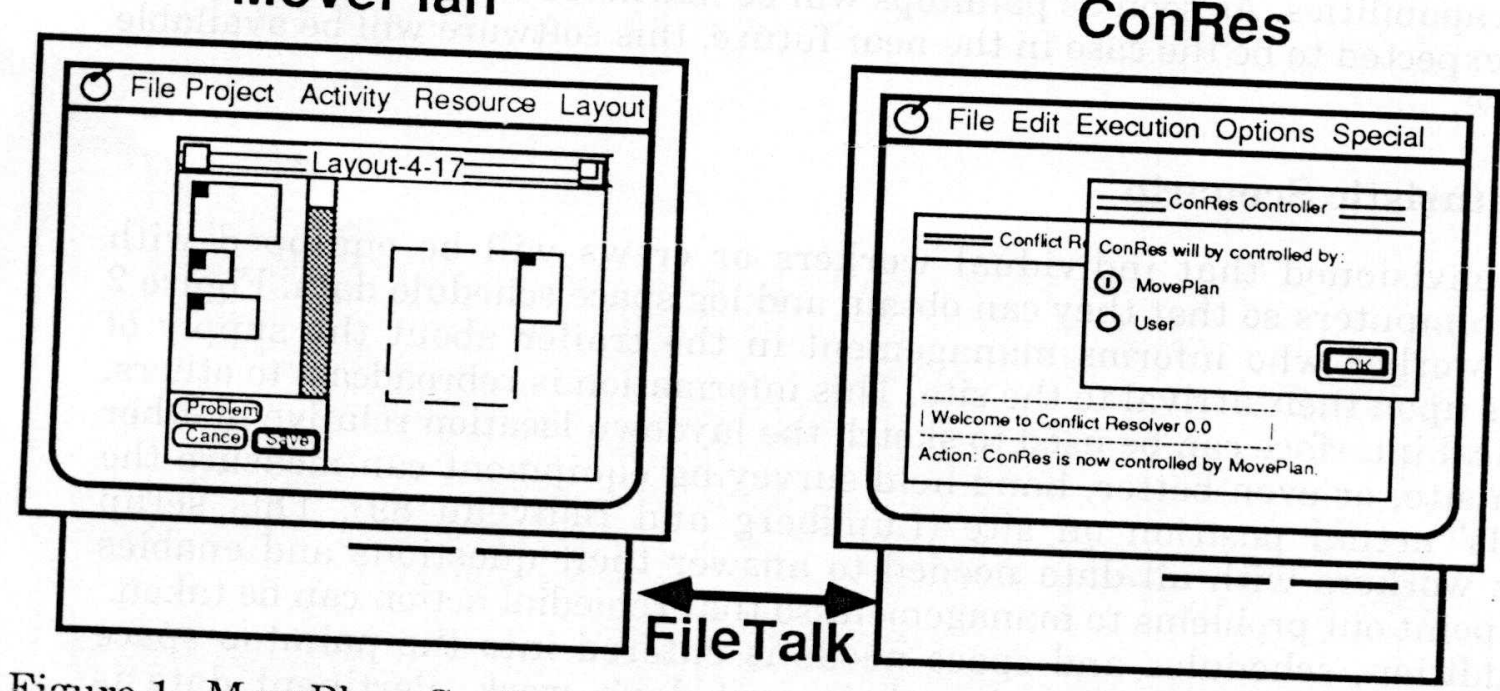

Figure 1. MovePlan-ConRes Interface 


\subsubsection{MovePlan-ConRes}

MovePlan has been loosely integrated with a space-schedule conflict resolver, named ConRes (Figure 1). ConRes opportunistically chooses one of several strategies for modifying a schedule so that space needs can be better accommodated in time intervals that are identified as problematic (Tommelein et al. 1993). Such strategies delay one or several activities to remove a resource from the current time frame while minimizing project delay or minimizing the level while minimizing project delay or maximizing the freed-up space. These strategies are by no means exhaustive, nor do they guarantee that a better schedule will be produced. Additional strategies for space schedule conflict resolution are being investigated.

\subsubsection{MoveSchedule}

The MoveSchedule program for space schedule construction tightly integrates and automates the space allocation and scheduling tasks. It is being developed by Zouein and Tommelein (1993). One key feature is that resources are modeled with lead times (the time period over which materials are on site before the activity starts) and safety stocks (the minimum amount of material on site that is maintained at all times until the material is no more needed; when a supply falls below the safety stock, it is replenished). The model also reflects resource depletion during the execution of an activity. It can thus represent just-in-time and just-in-case deliveries.

MoveSchedule will lend itself well to integration with a materials inventory control system, possibly tied to a bar coding system for tracking material arrival and placement in the permanent facility.

\subsection{Hardware Needs}

In 1983, the BRT pointed out that "Too little use is made of new, cheap microcomputers and software." (BRT 1983, p. 56). While this may still be true, one needs to look ahead and assess how the advent of palmtop computers is to change construction materials management. The above three programs all run on laptop computers. We plan to reimplement them in a language that efficiently uses palmtop capabilities. As soon as palmtops will be marketed at a reasonable price, which is expected to be the case in the near future, this software will be available for site use.

\subsection{Futuristic Scenario}

It is envisioned that individual workers or crews will be equipped with palmtop computers so that they can obtain and log space schedule data. Figure 2 shows a worker who informs management in the trailer about the supply of materials upon their arrival to the site. This information is rebroadcast to others. A graphical interface can be used to sketch the laydown location relative to other items on site, or even better, hand-held surveying equipment can measure the materials' actual position on site (Lundberg and Beliveau 89). This setup provides workers with all data needed to answer their questions and enables them to point out problems to management so that remedial action can be taken.

In addition, schedules and space needs is entered into the palmtop space scheduling tool by crews who plan their next day's work. Pertinent data is 
abstracted from it and broadcast to others who might detect interference problems or resource conflicts. Mediation software them helps them negotiate priorities.

This setup assumes that data can be entered and retrieved by all palmtops on site. Relatively powerful computers are therefore needed. How such a distributed communication system for on-site materials management will work within the current organizational structures and materials handling practice (e.g., assigning the appropriate responsibility and authority for adding, deleting, and modifying data) is a wide open area of research.

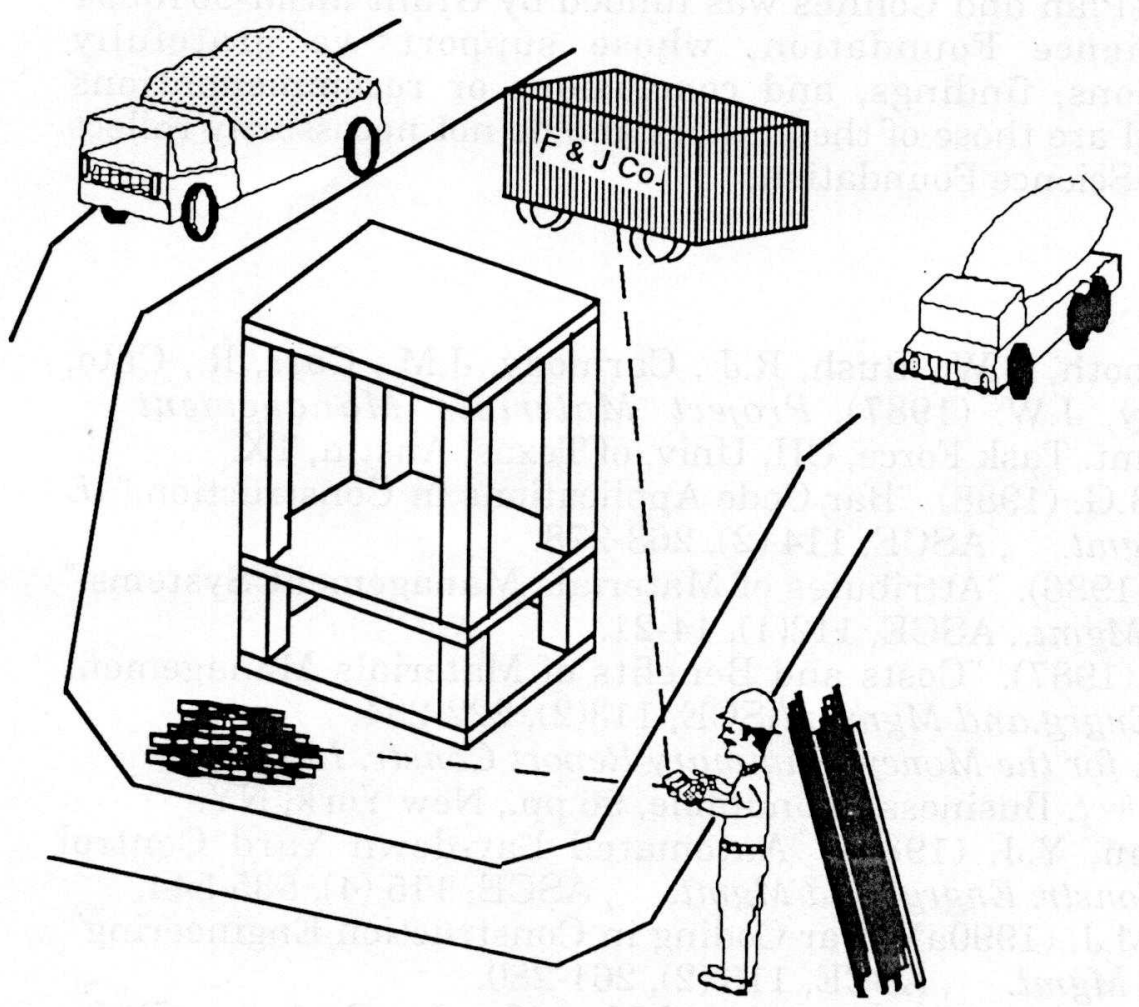

Figure 2. Futuristic Scenario for Use of Space Schedule Data

\section{SUMMARY}

Ten years ago, the Business Round Table concluded that "The material and equipment built into industrial and commercial facilities and power plants cost an estimated $\$ 82$ billion in 1979-some $60 \%$ of the total cost of those structures. But a CICE survey concludes that a significant part of the labor cost that went into that construction was wasted because materials and equipment were not available at the site when they were needed. That is the kind of foul-up modern management systems can minimize." (BRT 1983, pp. 29-30). At present, microcomputers and management techniques have not been exploited to the maximum of their potential. In the mean time, the technology has leaped forward and even more radical improvements to construction practice can be expected from the laptop and palmtop computer revolution. The desired use of such devices on site must therefore be researched, in anticipation of their wide-spread availability. Accordingly, the MovePlan, ConRes, and MoveSchedule models were 
briefly introduced, to show how space and schedule data can effectively be used in construction operations planning.

Issues pertaining to data collection for space scheduling, scheduling progress control, and data dissemination were discussed for obtaining an original and adjusting an existing space schedule if plan deviations become intolerable. A futuristic scenario illustrated the use of palmtops for space scheduling on site.

\section{ACKNOWLEDGMENTS}

The development of MovePlan and ConRes was funded by Grant MSM-9010394 from the National Science Foundation, whose support we gratefully acknowledge. Any opinions, findings, and conclusions or recommendations expressed in this material are those of the authors and do not necessarily reflect the views of the National Science Foundation.

\section{REFERENCES}

Bell, L.C., Bishop, R., Booth, F.W., Bush, R.J., Carmody, J.M., Carr, R., Cato, J.C., Dodd, G., Early, J.W. (1987). Project Materials Management Handbook. Matls. Mgmt. Task Force, CII, Univ. of Texas, Austin, TX.

Bell, L.C., McCullouch, B.G. (1988). "Bar Code Applications in Construction." J. Constr. Engrg. and Mgmt. , ASCE, 114 (2), 263-278.

Bell, L.C., Stukhart, G. (1986). "Attributes of Materials Management Systems." J. Constr. Engrg.and Mgmt., ASCE, 112(1), 14-21.

Bell, L.C., Stukhart, G. (1987). "Costs and Benefits of Materials Management Systems." J. Constr. Engrg. and Mgmt., ASCE, 113(2), 222-234.

BRT (1983). More Constr. for the Money. Summary Report Constr. Industry Cost Effectiveness Project. Business Roundtable, 96 pp., New York, NY.

Lundberg, E.J., Beliveau, Y.J. (1989). "Automated Lay-down Yard Control System-ALYC." J. Constr. Engrg. and Mgmt. , ASCE, 115 (4), 535-544.

Rasdorf, W.J., Herbert, M.J. (1990a). "Bar Coding in Construction Engineering". J. Constr. Engrg. and Mgmt. , ASCE, 116 (2), 261-280.

Rasdorf, W.J., Herbert, M.J. (1990b). "Automated Identification Systems-Focus on Bar Coding". J. Computing in Civil Engrg., ASCE, 4 (3), 279-296

Riley, D., Sanvido, V. (1992). "Site Material Management System." Proc. CIB 92 World Bldg. Congress, 596-597, Natl. Research Council Ottawa, Canada.

Sanvido, V., Paulson, B.C. (1991). "Site Analysis using Controller-Function Charts." J. Constr. Engrg. and Mgmt., ASCE, 117 (2), 226-241.

Tommelein, I.D., Castillo, J.G., Zouein, P.P. (1992). "Space-Time Characterization for Resource Mgmt. on Constr. Sites." Proc. 8th Conf. Comp. Civil Engrg. , ASCE, New York, NY, pp. 1042-1049.

Tommelein, I.D., Dzeng, R.Y., Zouein, P.P. (1993). "Exchanging Layout and Schedule Data in a Real-Time Distributed Environment." Proc. 5th Intl. Conf. Comp. in Civil and Bldg. Engrg., ASCE, New York, NY, June.

Tommelein, I.D., Zouein, P.P. (1993). "Interactive Dynamic Layout Planning." J. Constr. Engrg. and Mgmt. , ASCE, New York, NY, June 1993.

Zouein, P.P., Tommelein, I.D. (1993). "Space Schedule Construction." Proc. 5th Intl. Conf. Comp. in Civil and Bldg. Engrg., ASCE, New York, NY, June. 